\title{
An Enhanced K-Means Clustering Based on K- SVD_DWT Algorithm for Image Segmentation
}

\author{
M. Banu Priya, S. Jayasankari
}

\begin{abstract}
The complete nature of the image geared by various processes but image segmentation plays vital role. For object illustration, image analysis, visualization and image processing task the image is segmented into useful information by image segmentation. The image is segmented with respect to the opted scenario by image segmentation. The image measurements like texture, color and depth are considered by the segmentation. The plant disease can be spotted and classified in the field of agriculture and image segmentation is essential in image processing. Based on the morphological characteristics of plants, the diseases can be classified. Image segmentation is of importance within the field of image process. This work focuses on K-means Singular Value Decomposition (K-SVD) and Discrete Wavelet Transform (DWT) that is associated with Kmeans clustering for effectual image segmentation of leaf. Image segmentation is the basic pre-processing task to segregate the leaves in several image process applications. The most challenge in analyzing the plant images are locating and segmenting plants. Image segmentation accustomed to discover the objects and limits such as lines, curves, etc. in images. K-means cluster algorithmic program is wide employed in image segmentation to its machine simplicity. However, the clump results obtained from K-means heavily rely on the initial parameters. Mostly, these initial parameters are elite through hit and trial rule that ends up in inconsistency within the image segmentation results. In this work, an improved K-means cluster algorithmic program is projected for image segmentation, using a histogram based mostly initial parameter estimation procedure. To boot, the projected algorithmic program needs less user interaction to work out K-means data format parameters. Some experiments are conducted supported numerous gray images to check the projected approach. The experiment results show that the projected approach will improve the K-means based mostly image segmentation results.
\end{abstract}

Keywords: K-means clustering, Image Segmentation, Leaf disease, Histogram

\section{INTRODUCTION}

Among various techniques, the image procedure methods are getting terribly affordable and reliable day through day. There are numerous matters that may leads to critical diseases to the plants that have broken large cultivations subsequently for the economic system in nation get degrade. There are uncountable methods in use so as to look at the variety of illnesses for leaves by its starting periods. Thus to improve cultivation and also the financial system in national level, accurate and efficient identification of vegetation ailments was required. For greater exceptional and amount of vegetation in agricultural fields absolutely specific

Revised Manuscript Received on September 10, 2019.

M. Banu priya, Ph. D Research Scholar (Full time), Department of Computer Science, P.K.R Arts College for Women (Autonomous), Affiliated to Bharathiar University, Reaccredited With 'A' Grade by

Dr. S. Jayasankari, Associate Professor in Computer Science, P.K.R Arts College for Women (Autonomous), Affiliated to Bharathiar University, Reaccredited With 'A' Grade by NACC, Gobichettipalayam, Tamil Nadu, India. NACC, Gobichettipalayam, Tamil Nadu, India.

techniques are deliberate with the aid of one-of-a-kind analyzers by the support of DIP process for speedy and right plant life sickness identification [3]. To avoid wasting time, labors, efforts and needs in serious pesticides leads to requirement for quick \& right ailments identification in agriculture field. While ailments was identifies in first groundwork then avoided consequently, then massive downflow may also be avoided.

Denoising may be a noteworthy feature of the image process. Within the most up-to-date decades, some denoising strategies are planned. One category of such strategies contains those that take good thing about the examination of the image during a (repetitive) frame. As an example, during this set, the brink price of the image coefficients may be denoted in an orthonormal basis, like the trigonometric function basis, a rippling basis, or a curvelet basis. The efficiency of those strategies comes from the fact that the images can be in moderation approximated in those dictionaries. It's divided into three steps: a) slight writing step, wherein, the preliminary dictionary, slight approximations are used for the computation of all patches (with a tough and rapid size) of the picture; b) dictionary replace, anywhere updation is carried out in the dictionary in the sort of style that the same old of the thin approximations is accelerated; and next, c) reconstruction step that recovers the denoised image from the gathering of denoised patches and d) the algorithmic rule carries out $\mathrm{K}$ iterations of steps a and b. In some other category, one would possibly embody techniques that make the maximum of the nonneighborhood similarity of patches within the image. During this type, the alternatives may be involved that try and recover the maximum systems of the sign through employing a dictionary (which on the whole includes a probably redundant set of generators). The K-SVD primarily based denoising algorithmic rule merges a few thoughts returning from those classes, paving the manner of dictionary gaining knowledge. The K-SVD technique also may be helpful in alternative image responsibilities, like non-uniform denoising and demosaicing. Indeed, the potency of the dictionary is encoded via practical standards which are optimized taking the income of the nonneighborhood similarities of the image. The orthogonal matching pursuit and the matching pursuit algorithmic rule are of this type. The variational techniques are the kind of second class of denoising algorithms. Among them, the total variant (TV) denoising is most famous anywhere and the selected regularity version is that the set of functions of finite versions.

The previous work was done on the preprocessing step,

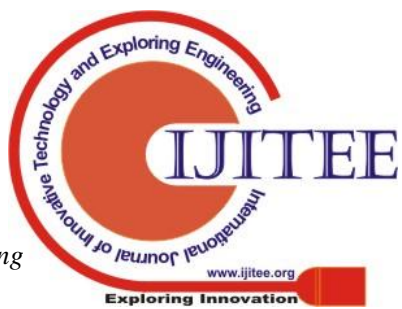


wherever the denoising technique is enabled to attenuate the noise for affording consistent ends up in the realm of image process. Here, the denoising feature is performed by examining the element ratios of the healthy leaves and therefore the pixel values of an exaggerated leaf image are compared with the healthy leaf. The tactic is examined with the assistance of K-SVD_DWT method. The method is important for attaining faster and precise recognition of leaf diseases at the start stage. Future stage is image segmentation. Image segmentation techniques play a key role and function of the inspiration for varied sorts of image process. Recently, image segmentation is trending where the images are segmented with meaning and processing the basic units. The required object can be accurately segmented and investigated by image segmentation techniques. The past theory says that there are feature and model oriented segmentation. The backgrounds as well as different background objects are based on segmentation object.

Image segmentation is applied to cluster pixels into salient image regions, like regions adore objects of the image. These regions may be thought of undiversified in keeping with some characteristics, like gray level, motion, texture, and shape. Image segmentation is a vital initial step in image process and is employed in varied field on particle identification on remote pictures, biomedical, control systems, facial authentication and machine vision. Image segmentation may be achieved by employing a heap of techniques, which may be generally classified into region and edge-based mostly techniques. Edge-based mostly techniques represent the pixels within the image having sharp intensity variations. Generally, there may be broken incomplete edges and there's not a transparent separation between the objects within the image. On the opposite hand, region-based mostly techniques are based upon the gathering of pixels having similar options, which may be gray-level intensity data or color image elements.

Recently, a lot of and more researchers specialize in the region based mostly techniques victimization cluster ways, as a result of its simplicity. Cluster is one among the necessary and generally used techniques and is widely employed in engineering fields like pattern recognition, image process, system modeling, and data processing. In cluster techniques, the previous information of the formatting parameter estimate, i.e., variety of clusters and therefore the several initial cluster centers is needed, that is usually input by the user or every which way chosen from the input file. The impact of those parameters upon the ultimate image segmentation is big, and variation of those parameters produces fluctuations within the final results. One among the foremost well-known and quickest cluster techniques is that the K-means technique. K-means could be an onerous cluster algorithmic rule, within which every element solely belongs to one cluster center. K-means recomputes every one of the new centers by averaging the element intensities appointed to the previous cluster centers. During this work, to specialize in the region based mostly image segmentation victimization K-means cluster ways as a result of its simplicity and high potency. However, there are some shortcomings of the K-means based mostly ways that ought to be solved, for instance, it's sensitive to outliers

and additionally, its greedy nature makes it sensitive to formatting parameters.

The planned rule of the decomposition of images contains function of image based segmentation with K-means clustering set of rules. This work describes the method of ripple redesign utilized as alternatives extraction combined with each pixels of image and mixing this approach with the $\mathrm{K}$-means agglomeration method. Individual techniques are validated for everyday images and applied for the manner of elite real time plant leaf images. The strategy represented is employed for the outline of the overall machine outstanding picture reconstruction. A deliberate approach that creates use of wavelets and K-means clustering is carried out for leaf snapshots. The ripple method and the K-means clustering are blended in this method to provide better accuracy results. For the decomposition of the image, a specific part of the work is explained concerning the decomposition and reconstruction matrices. Through wavelets, excessive image is extracted and to boost the additional information decomposition is carried out, that offers expanded side information with ripple alternatives like entropy and energy.

\section{RELATED WORK}

A leaf image is split into 4 clusters. Then Extraction of units of alternatives by way of coincidence technique to research disease leaf's content become completed. Identification \& class for illnesses type's ANNs had been achieved. K means rules play the segmentation technique for the duration of this work [1]. During this approach, ANN was utilized to find intention in affected leaf routinely. Once the leaf is affected by the sickness then there'll be one or additional clusters that may have the ailment. Hiary projected automatic and smart decision to find out and classify the leaf's sicknesses routinely with efficient and precision way. By processing it pixels from image are categorized to idea in collection of options into the $\mathrm{K}$ range of classes. The accuracy is $94 \%$ accuracy and moreover, $98 \%$ computational time is attained.

The image which was initial is non-heritable therefore the vector of image median filter is hired for initial process the crop leaves image. Hence projected techniques observe three steps: Pre-processing, Segmentation and math evaluation. Throughout preprocessing, data approach is hired instead of the historic image enrichment approach. At the end the level of affected region in crop sickness is noticed [2]. Then traditional and affected areas are segmental via implemented math pattern recognition. Then a quantitative percentage of the conventional and affected area is calculated. With the usage of improved vector median, the higher edges are performed. Output result of ailment is chosen on the idea of infected and traditional difference in color area. Tian projected the course in which expansion and grading time is reduced compared to the manual device.

Researchers pointed for quicker identification for plants sicknesses and contrasted with a number of the presently used techniques [3]. 
The images of the plant are snapped via the digital cam then it was connected with PC, Pre-process of image and Extracting alternatives. SVM is then used for categorization. Image is expanded at some stage in this step. Last it's aforesaid that the projected gadget is dependable for faster pest's detection. The projected technique has Acquisition of the image. Differing types of tormentor collectively with whiteflies, aphids, etc affected that vegetation in numerous methods. Mundada selected certain types of crops together with cucumber, rose, tomato, capsicum, etc and cultivated them in exceeding farmland. The classification technique is completed with the assistance of gray coincidence and region homes.

As a result of the green-colored area approach, those are the healthful additives of the leaves. These values are computed for these pixels. Ananthi said that the plant diseases can also be of three types such as viral, fungus and microorganism [4]. The RGB photo is born-again into HSI (Hue Saturation Intensity) format. Good classifiers in short period, the RGB pixels were made to zero. With the given approach initial images are taken by using the camera. The RGB photos of all the leaves samples are amassed. Image procedure techniques are applied to investigate and acquire a few essential alternatives. For protecting of green pixels preliminary of all green pixels are known. The planned technique is used for quick detection and type of plant's illnesses. Masking of inexperienced pixels is finished. It removes the cloaked green pixels. Then principally green pixels are cloaked based usually upon specific threshold values.

The method of pest's segmentation turned into commenced by using feature green colored pixels depending upon completely exceptional threshold values. Currently, for RGB pests photographs coloration transformation structure is turned into normal. Initially, they noted the diverse present-day researches and in order that they noted that the blended result of the strategies (K-Means clustering program and correspondence) for automated identification of pests [5]. The authors have tested ten absolutely specific pests. Fina used a K-Means cluster algorithmic application and to identify the pests. For pest's segmentation, k-way clustering is used. The deliberate approach for pest's detection is split into two steps: (1) Detection and (2) Recognition in the initial part of the algorithmic application, pixels are captured first and so those captured footage are loaded because of the filtering created snapshots. Color residence transformation turned into implementation. The Red and Blue color factors of the pixels of the fastened threshold charges are appointed zero range to ignore such values.

The outcomes of fungus infections is ascertained in definitely various culmination such as mangoes, grapes and pomegranates. Pujari endorsed the math procedures are observed first and so to categorize the fungus sicknesses [6]. Affected data's due to fungous illnesses had taken initially and so categorized based on acute function of cited sicknesses such as ordinary, inflamed, and mild infected and extreme infected. Totally one of a kind applied math alternatives like block-smart, grey Level Co prevalence Matrix, grey Level Run-duration Matrix are extracted from these snapshots. Geometer is employed to categorize those snapshots, as every day, element inflamed, reasonably

inflamed and significantly infected. The classification accuracy has been improved to $94.085 \%$.

\section{MATERIALS AND METHODS}

\subsection{Denoising Process}

The process is executed by assimilating each K-SVD and DWT. The most purpose of this analysis is to make complete dictionary system by enhancing K-SVD_DWT for image denoising, still, as mistreatment this algorithmic rule to realize quicker image denoising method than the standard $\mathrm{K}-\mathrm{SVD}$. The dataset is predicated on classifying the leaves into one in all the subsequent types: 'Alternaria Alternate', 'Anthracnose', 'Bacterial Blight', 'Cercospora Leaf Spot' and 'Healthy Leaves'.

To deal with this downside, the concept would be to use the K-SVD algorithmic rule to each channel R, G, and B and used one after the other. This resolution has not been attempted because of the brand new proposition seems even brighter. It's time to provide the method to adapt the grayscale algorithmic rule to shade snapshots. Another proposal could be to use a foremost component analysis on channels RGB, which would possibly uncorrelate them, and so to apply the proposal throughout this extra relevant setting. They're to the real truth that in natural images, there may be a completely vital correlation among channels. This naive resolution offers shade artifacts.

So as to get the colors properly, the algorithmic rule antecedently delineated are applied on column vectors that are the concatenation of the R, G, and B values. During this manner, the algorithmic rule can higher update the dictionary, as a result of its ready to learn correlations that exist between color channels.

The Orthogonal Matching Pursuit (OMP) algorithmic rule could be a greedy algorithm which tries to search out a distributed illustration of a proof given a selected dictionary. The algorithmic rule tries to search out the simplest basis vectors (atoms) iteratively specified in every iteration the error in the illustration is reduced. This achieved selectively of that atom from the wordbook that has the most important absolute projection on the error vector. This basically implies that we tend to choose that atom that adds the utmost info and thence maximally reduces the error in reconstruction.

The K-SVD algorithmic rule tries to attenuate the value perform iteratively, by 1st finding secret writing for the signals in question mistreatment the OMP algorithmic rule using the initial estimate of the dictionary. This secret writing is sought-after specified it minimizes the error in illustration, and at identical time maintains a scantiness constraint. Once this distributed secret writing stage is completed, the algorithmic rule yield to update the atoms of the dictionary, one atom at a time, specified the error term is additional reduced. Continuing in such unvaried technique the algorithmic rule reduces, or at the worst maintains, the error of illustration within the iteration.

Having outlined a general read of the steps of the 
algorithmic rule, tend to proceed to detail the steps and also the mathematical basis behind the tactic.

\section{$3.2 \mathrm{~K}$-means Clustering}

An image is taken care of supported key-word (metadata) or it's content (description). The comparably image features were sorted for make a cluster by way of a challenge really worth to each characteristic. The strategies and algorithms which can be utilized from parameters like information, pattern recognition, signal process, and so on. A cluster is for a fixed of items which can be "similar" between them and are "distinctive" to the object to opportunity clusters. Clusters supported the optimization of associate overall lifestyles may be an essential approach explored because of the first days of pattern popularity. Clustering can be a method of organizing the items into teams supported its attributes. In content-based clustering, content material implies to textures, shapes or the alternative data may be inheritable from the image itself. The important popularity is K-means clustering. In keyword-based clustering, a key-word can be a style of font that describes regarding the photo key-word of a photograph and refers to its completely unique alternatives.

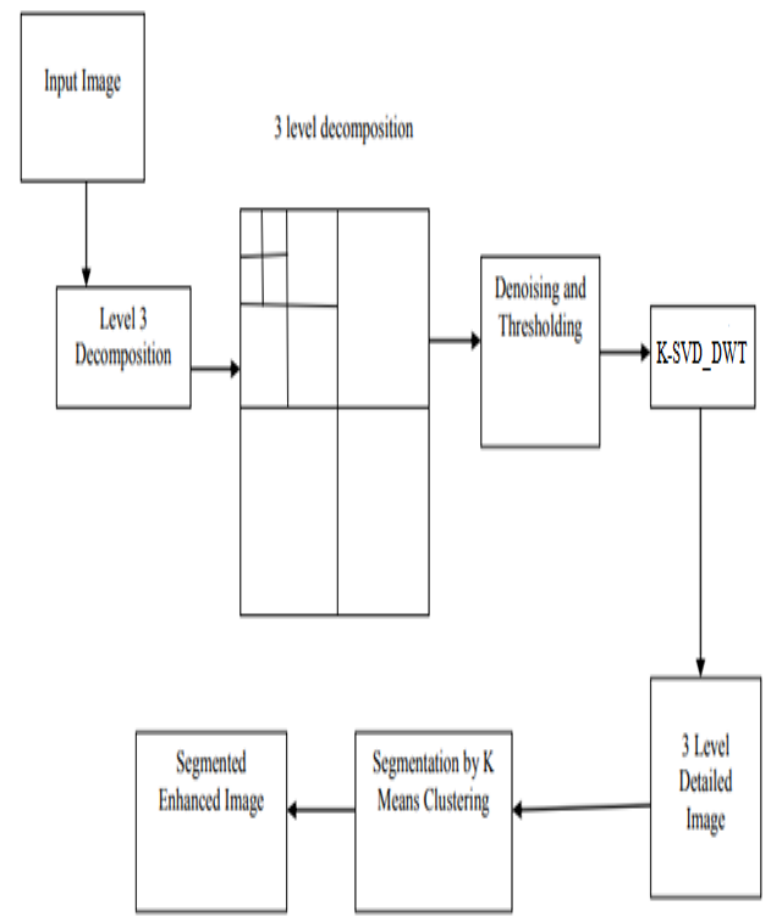

Figure 1: Proposed Framework

The size of the centroids is the same due to the dimension of the records vectors. In a supervised clustering technique, the grouping is completed in step with user comments. Every pixel is appointed for cluster which supports nearest to the pixel, that was set by the geometer distance. In the beginning, the centroids for determined clusters was initialized haphazardly. In the end, the pixels are clustered; the implications of every cluster is recalculated. In K-means algorithmic program information vectors are looked after right into a predefined variety of clusters. The center of mass needs to be chosen to be distinctive and it has to reduce the entire distance some of the clusters. Each commonplace technique used with efficiency for pattern

supervised and unsupervised cluster strategies are utilized in photo segmentation. In an unsupervised cluster, the image with excessive alternatives is completely specific in phrases of linguistics. This method is continual to no vital changes result for every cluster mean or for a few mounted levels of iterations. In K-means cluster a center of the mass vector is computed for each cluster. The deliberate framework is given in figure 1 .

The k-means algorithmic rule is employed as an area find algorithm which contributes an settled world optimization technique. This system proceeds in progressive approach of trying to optimally embody one new cluster center at every stage rather than indiscriminately choosing beginning worth for all clusters. Additionally, to resolve a cluster downside with k clusters the tactic return as follows.

1: Algorithmic rule initiate by single cluster $(k=1)$ then center of cluster respective to the center of mass of the information set $\mathrm{X}$.

2: Stimualets $\mathrm{N}$ number of iteration in $\mathrm{k}$-means to seek out 2 clusters $(\mathrm{k}=2)$ once starting place of the cluster centers: For $\mathrm{k}=1$, the primary cluster center is continually placed at the optimum place. Then next center of iteration $n$ is stored in the place of the information purpose $x_{n}$ ( $n$ $1, \ldots \ldots, \mathrm{N})$. The most effective answer is attained once $\mathrm{N}$ iteration of the k-means algorithmic rule.

3: For k-1 cluster downside, the ultimate answer is denoted by $\left(\mathrm{c}_{1}, \mathrm{c}_{2}, \ldots, \mathrm{C}_{\mathrm{K}-1}\right)$. The algorithmic rule performs $\mathrm{N}$ iteration of the $\mathrm{k}$-means algorithm with starting place $\left(\mathrm{c}_{1}, \mathrm{c}_{2}, \ldots, \mathrm{C}_{\mathrm{K}-1}, \mathrm{x}_{\mathrm{n}}\right)$, wherever $\mathrm{n}$ varies from one to $\mathrm{N}$ to seek out an answer for the k-clustering downside. The most effective solution obtained during this method is taken into account because of the racial extermination.

The methodology is planned in five steps and it is diagrammatically given. The steps are shortly described:

1. K-SVD_DWT was utilized to input image of leaf to get wavelet decomposed image leading to 12 sub bands. Of these sub bands represent approximation, horizontal, vertical and diagonal parts within the kind of coefficients, severally. LL sub band contains low level and therefore the alternative 3 (LH, HL, HH) contain high-level details.

2. Denoise the decomposed leaf image exploitation average filter and global thresholding. Otsu's model is applied for separating the background and therefore the form of leaf is extracted.

3. Set approximation coefficients in LL up to zero and apply inverse wavelet transform to get a high pass image from the remaining (horizontal, vertical and diagonal) sub bands. It has a tendency to decision the resultant image level-3 (L3) detail image.

4. Add L3 to the first image to urge a sharpened image.

5. Apply the K-means that cluster algorithmic rule, as given in step [3], to segment the images that partition the information set into the best sort of clusters. Same information factors belong to as a minimum one cluster and absolutely distinct statistics factors belong to one of kind clusters. This algorithmic rule owed for variability in cluster shapes, cluster densities and therefore the form of 
information points in every one of the subsets. The K-means has a disadvantage that it can't work simply in case of screeching plant leaf snapshots. Therefore, the mixture of KSVD_DWT and K-means that offers higher results as wavelets are strong to noise and this combination additionally helped to get rid of in-homogeneity and artifacts created in plant leaf imaging.

6. The sting data is corrected exploitation Sobel edge detector and at last the improved image is obtained.

The K-SVD algorithm tries to decrease the cost feature iteratively, by using locating a coding for the signals. Additionally, their multi resolution lets in inspecting the images at numerous scales and orientations. The software of the K-SVD_DWT algorithm is used to denoising of images. Over-decomposition has to be prevented, as a result of due to the fact the decomposition ranges boom, there may be a wonderful hazard that lower frequencies grow to be a locality of detail components. The multi-decision property offers facts concerning various excessive-frequency parts at completely exceptional degrees of decomposition. KSVD_DWT is implemented to plant leaf images because of wavelets that offer frequency data additionally as time-space localization. Before that may be addressed, the method may be scaled up to address photo denoising of arbitrary sizes.
This could limit the less stage of decomposition because of lower frequencies and that can grow to be part of excessive images and scale back powerful element in an image.

\section{RESULTS AND DISCUSSION}

The rule segments a picture per similar options among pixels. The picture texture options are extracted in the primary step. Next step is forming the clusters by K-means rule. The result is the idea for knowledge grouping. Steps for the K-means rule are as follows:

Step 1: Choose the cluster center.

Step 2: Confirm the options for each constituent.

Step 3: The similarity is outlined among the cluster options and its centre (Euclidean Distance).

Step 4: The new cluster centre is computed.

Step 5: The $3^{\text {rd }}$ and $4^{\text {th }}$ steps have to be repeated till the centre of cluster stops dynamically.

The distinction, coarseness and the directivity for every constituent is divided by the rule into 2 teams ( 0 or 1 ) by the $\mathrm{K}$-means algorithms proposed in this work. Once the denoising method is over then the image is loaded and it's shown in figure 2 and the contrasted image is shown in figure 3

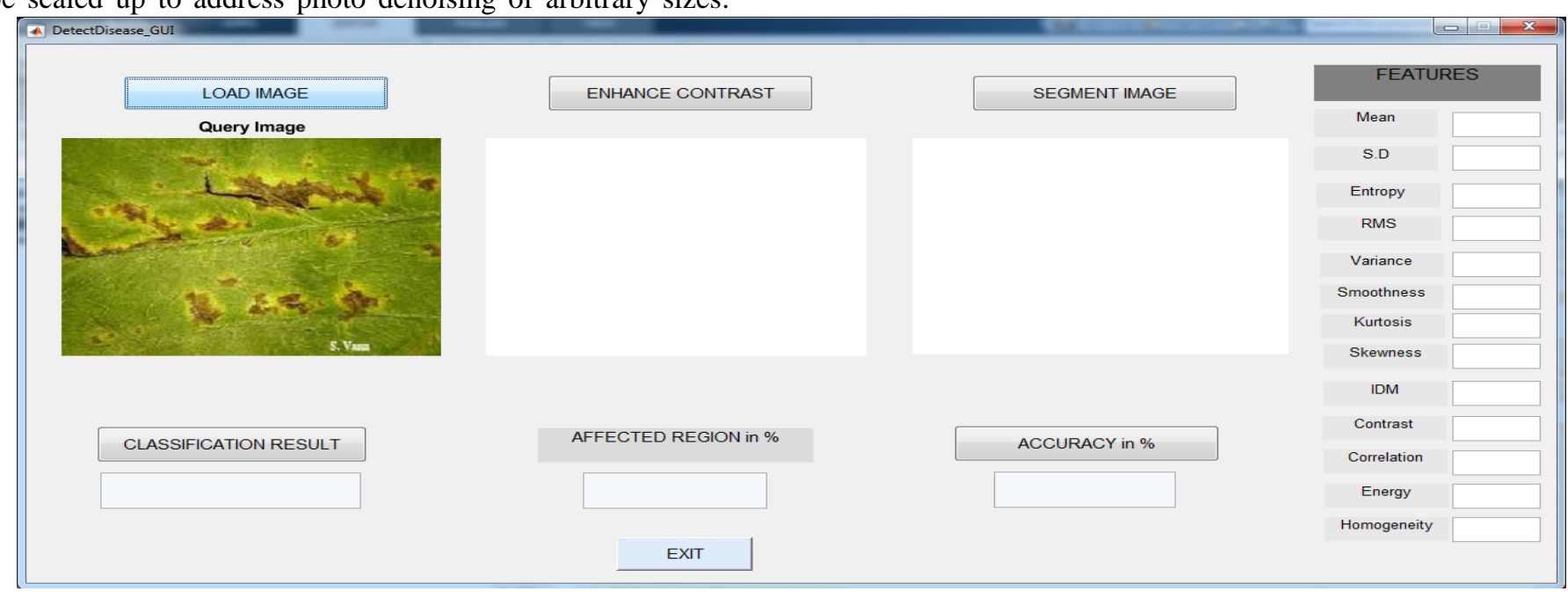

Figure 2: Query Image

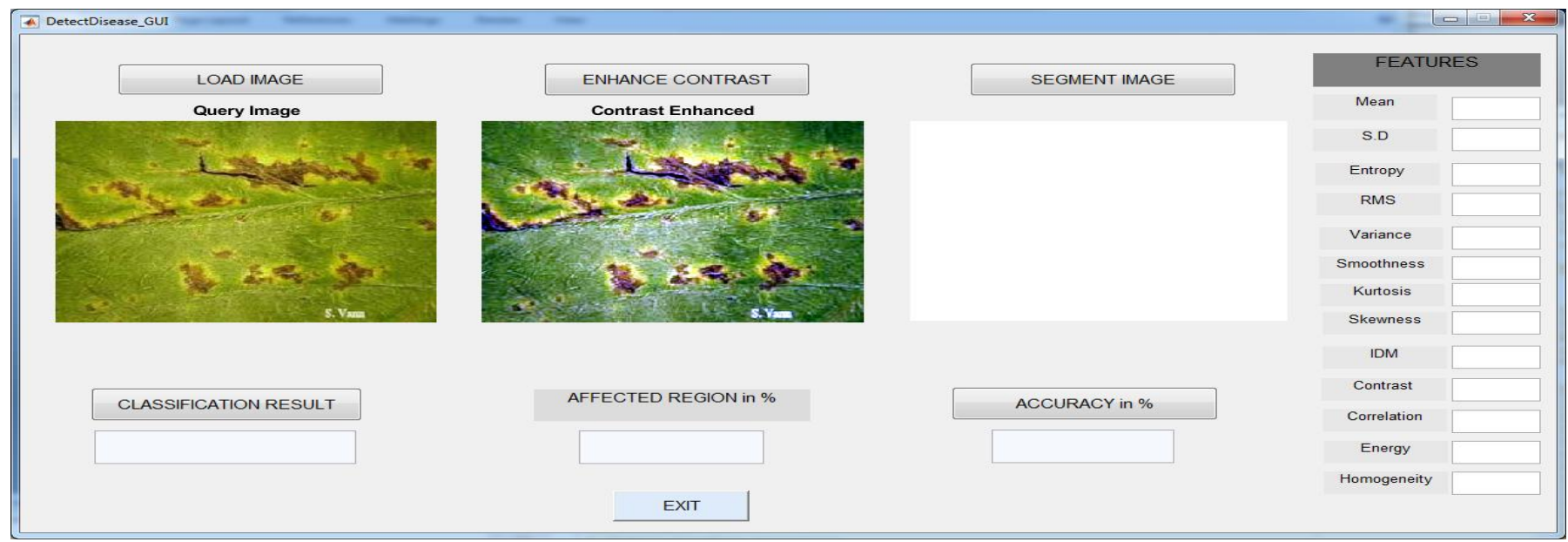

Figure 3: Contrast Image 


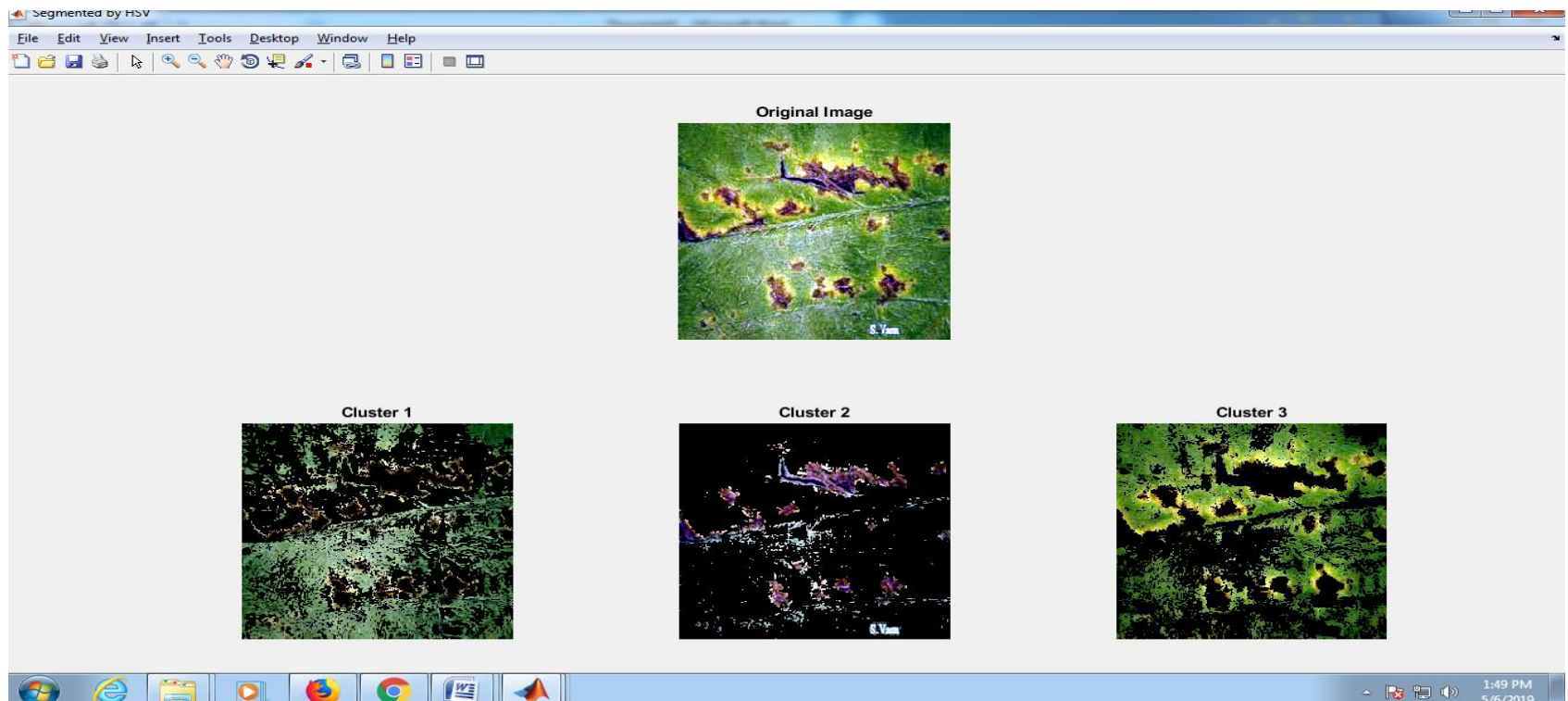

\section{Figure 4: Cluster Formation}

The segmentation of the image has been victimized by the $\mathrm{K}$-means formula. The enhancement of the cluster is by presuming neighboring pixels that have a high likelihood of declining into an equivalent cluster. In the image plane, the pixels are observed by the applications of image segmentation. The image is segregated into $\mathrm{k}$ number of non-continuous sections by K-means segmentation. The Kmeans segmentation formula is given as follows:
Step 1: The K value is chosen.

Step 2: The K-means formula is applied.

Step 3: The associated formula is applied.

Step 4: The part with any size can be merged with the adjacent element's threshold.

Step 5: The background image's Segmentation.

The clusters are performed and it's shown in figure 4

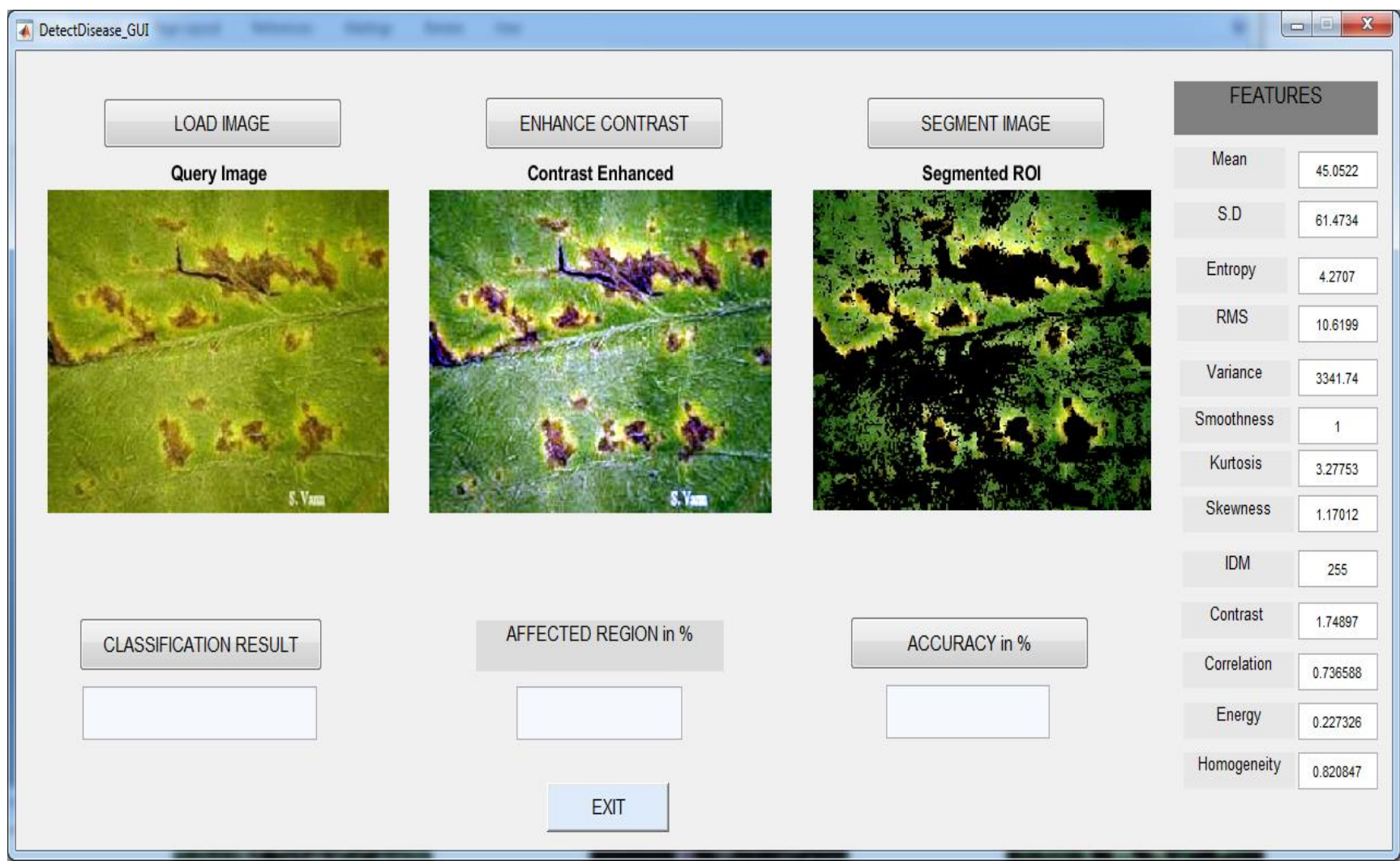

Figure 5: Segmented ROI

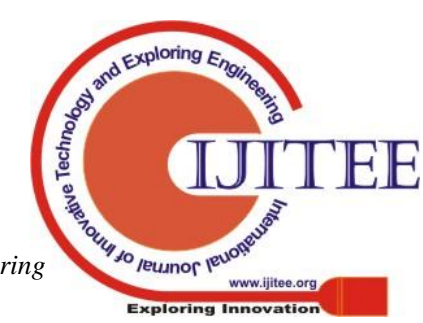


Segmentation is involved with two phases and it plays a major role: The first phase is involved with leaf separation from different encircling components of the image. Then the second phase is connected with the region of interest which is said to be ROI segmentation. These two phases connectedly removes the leaf's affected areas. The distinction of color is given by disease symptoms from other areas. The green pixel masking will results in ROI segmentation. The affected properties are extracted and it is the main feature of segmentation from the ROI. The style characterization option helps the human to observe from the disease classification results.

The knowledge can be characterized by utilizing the machine learning classifier. The segmental ROI is given in figure 5.The role of segmentation is to extract the picture's texture options rule and to classify the similar feature texture regions. The result shows that affected regions are segregated in various conditions. The segmentation is accurately performed by observing the object.

\section{CONCLUSION}

The detection of leaf disease in machine learning has gained incredible thrust in the field of agriculture. The higher quality measurements are attributed which includes various techniques and helps to combine the different image sources into the dataset. Hence the image segmentation algorithm is projected which serves as the basis for volume computation. There are several advantages when compared to traditional techniques. Firstly, within the K-means technique, the tactic of determinant $\mathrm{K}$ is optimized, and therefore the loop is employed to match the number of connected domains that meet the necessities within the final step, and after they are equal, the $\mathrm{K}$ value is chosen properly. This work mentioned on combination of $\mathrm{K}$ SVD_DWT and related to the K-means that cluster for economical plant leaf image segmentation. The planned approach is verified with real time plant leaf knowledge base. The results of the planned approach provide higher convergence in comparison to traditional segmentation technique.

\section{REFERENCES}

1) H.Al-Hiary, S. Bani-Ahmad, M.Reyalat, M.Braik \& Z.AlRahamneh, "Fast and Accurate Detection and Classification of Plant Diseases", International Journal of Computer Applications, Vol. 17, No.1, pp. 31-38.March 2011.

2) Y. Tian, L. Wang and Q. Zhou , "Grading method of Crop disease based on Image Processing", Computer and computing technologies in agriculture 427-433, 2011.

3) R.G. Mundada, Dr. V.V. Gohokar, "Detection and classification of Pests in Green House using Image Processing”, IOSR Journal of Electronics and Communication Engineering (IOSR-JECE) Vol. 5, Issue 6, PP 57-63, 2013.

4) S. Ananthi \& S.V. Varthini, "Detection and Classification of Plant Leaf Diseases", International Journal of Research in Engineering \& Applied Sciences, Vol. 2, Issue 2, pp.763-773, February 2012.

5) F. Fina, P. Birch, R. Young, J. Obu, B. Faithpraise and C. Chatwin, "Automatic Plant Pest detection and recognition using k-means clustering algorithm and correspondence filters", International Journal of
Advanced Biotechnology and Research, Vol. 4, Issue 2 pp 189-199, 2013.

6) J. D.Pujari, R. Yakkundimath \& A.S.Byadgi, "Statistical Methods for Quantitatively Detecting Fungal Disease from Fruits" Images", International Journal of Intelligent Systems \& Applications in Engineering Advanced Technology \& Science, vol.1 no.4, pp.60-67, 12th Dec2013 